\title{
Non Adiabatic Evolution of Elliptical Galaxies by Dynamical Friction
}

\author{
S.E. Arena ${ }^{1}$, G. Bertin ${ }^{1}$, T. Liseikina ${ }^{2}$, and F. Pegoraro ${ }^{3}$ \\ ${ }^{1}$ Università degli Studi di Milano, Dip. di Fisica, via Celoria 16, 20133 Milano, Italy \\ (Serena.Arena@unimi.it, Giuseppe.Bertin@unimi.it) \\ ${ }^{2}$ Institute of Computational Technologies, SD RAS, Novosibirsk, Russia (tatianavl@nga.ru) \\ ${ }^{3}$ Università di Pisa, Dip. di Fisica, largo Pontecorvo 3, 56100 Pisa, Italy (pegoraro@df.unipi.it)
}

\begin{abstract}
Many astrophysical problems, ranging from structure formation in cosmology to dynamics of elliptical galaxies, refer to slow processes of evolution of essentially collisionless self-gravitating systems. To determine the relevant quasi-equilibrium configuration at time $t$ from given initial conditions, such slow evolution is often approximated in terms of adiabatic evolution. Here we focus on the slow process of evolution induced by dynamical friction of a spherical stellar system (the host galaxy) on a minority component of "satellites" (distributed in a spherical shell), to determine to what extent an adiabatic description might be applied.
\end{abstract}

Two experiments of slow evolution We use N-body simulations to follow the evolution of the host galaxy interacting with the "satellites" of the spherical shell. We perform two groups of simulations: (1) the shell is dragged in by dynamical friction, (2) the shell is evolved adiabatically (in the absence of dynamical friction). We compare the evolution in the two cases both at the macroscopic (density and anisotropy profiles) and at the microscopic level (single particle total energy $E$, angular momentum $J$ and radial action $I$ ). We also examine the behavior of two classes of galaxy models characterized by significantly different density and pressure anisotropy profiles.

Results We demonstrate that the evolution driven by dynamical friction is quantitatively and also qualitatively different from the adiabatic case. In the dynamical friction case the final density profile of the host galaxy is shallower in the inner region and broader in the outer parts, in contrast with the central sharpening and the external lowering observed in the adiabatic case. Discrepancies are significant also at the microscopic level where, in the evolution induced by dynamical friction, more than $70 \%$ of the galaxy particles change their value of $J$ and $I$; these changes are not present in the adiabatic evolution where, within the numerical resolution of the code, the two quantities are conserved by all particles. Finally, the two classes of galaxy models considered in this investigation exhibit generally similar trends in evolution, with one exception: concentrated models reach a final total density profile, in the inner region, shallower than the initial one, while galaxy models with a broad core show the opposite behavior.

Conclusions The evolution of elliptical galaxies induced by dynamical friction is a slow process but it is not adiabatic. In addition, the evolution of the total density profile of the host stellar system depends on the concentration of the galaxy model. In conclusion, the results of our investigation should be taken as a warning against the indiscriminate use of adiabatic growth prescriptions in studies of the structure of galaxies.

\section{Reference}

Arena, S.E., Bertin, G., Liseikina, T., \& Pegoraro, F. 2006, A\&̊A, 453, 9. 